\title{
3D DATA INTERPRETATION USING TREATISES GEOMETRIC RULES TO BUILT COFFERED DOMES
}

\author{
M. Capone ${ }^{1}$, E. Lanzara ${ }^{1}$ \\ ${ }^{1}$ UNINA, DIARC Department of Architecture of Naples, Italy - (mara.capone, emanuela.lanzara)@unina.it
}

Commission II, WG II/8

KEY WORDS: Generative modeling, Coffered Domes, Parametric modeling, S. Giovanni Maggiore, Vannini, Apollonio theorem, Archimedes theorem, HBIM.

\begin{abstract}
:
The contribution is part of a research that aims to address the problems of knowledge, interpretation and documentation of coffered domes geometry. The main question is to define the relationships between the coffer shape, the layout used for coffers distribution on dome surface and different kind of surfaces. With regard to coffered domes we have analyzed the methods illustrated by Francesco Milizia, Giuseppe Vannini and some historical surveys. We have grouped coffered domes in relation to grid geometry and to coffer shape. We have defined three different ways to distribute the coffers in relation to different grid layout: grid composed by 2D lines (meridians and parallels), grid composed by 3D lines on surface (lattice of rhumb lines) or coffers distribution between ribs. We have analyzed each of them and we have defined algorithmic models in relation to spherical domes. The main goal of our research is to study what's different in not spherical domes, such as policentrical domes, ellipsoidal domes or ovoidal domes, generated using curves network. We have compared computational models based on treatises rules with particular case studies. This comparison allows us to do a critical analysis based on geometric rules. From a methodological point of view we have built a parametric model able to connect the different processes, using the same parameters. By comparing this model with point clouds, it is possible to evaluate analogies or identify new rules that will be used to develop a more complex parametric model based on surveys.
\end{abstract}

\section{INTRODUCTION}

The contribution is part of a research that aims to address the problems of knowledge, interpretation and documentation of coffered domes geometry.

In our research we analyzed some of the geometric methods described in Treatises with the aim to compare the theoretical model with the real based model. With regard to coffered domes we have analyzed the methods illustrated by Francesco Milizia in "Principi di Architettura Civile", by Giuseppe Vannini in "Elementi di Architettura Civile" and some historical surveys. We are looking for other examples and we are going to use other sources such as historical surveys.

We have grouped coffered domes in relation to grid geometry and to coffer shape. When you overlap a grid on inside surface of a dome, you can create a series recessed areas, known as coffers. They can have structural or ornamental purposes. The most famous coffered dome is the dome of Pantheon. There are many researches that analyzes various hypotheses about the geometric pattern and setting out of the coffers in the dome of revolution, most of them refer to the study of the coffered dome of the Pantheon(Fernández-Cabo, 2013).

We have identified a set of problems to solve for the design and construction of a coffered dome to define the geometric rules, appropriately choose the parameters to construct parametric models using computational algorithmic modeling.

Some of the main parameters are:

- number of meridian divisions (number of coffers in horizontal planes);

- number of coffer rows;

- Proportion between the meridian rib width and the coffers width;

- Proportion between height and width of the starting coffer and consecutive coffers.

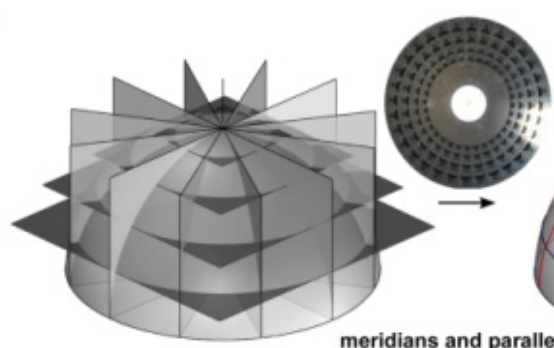

WEB GEOMETRY
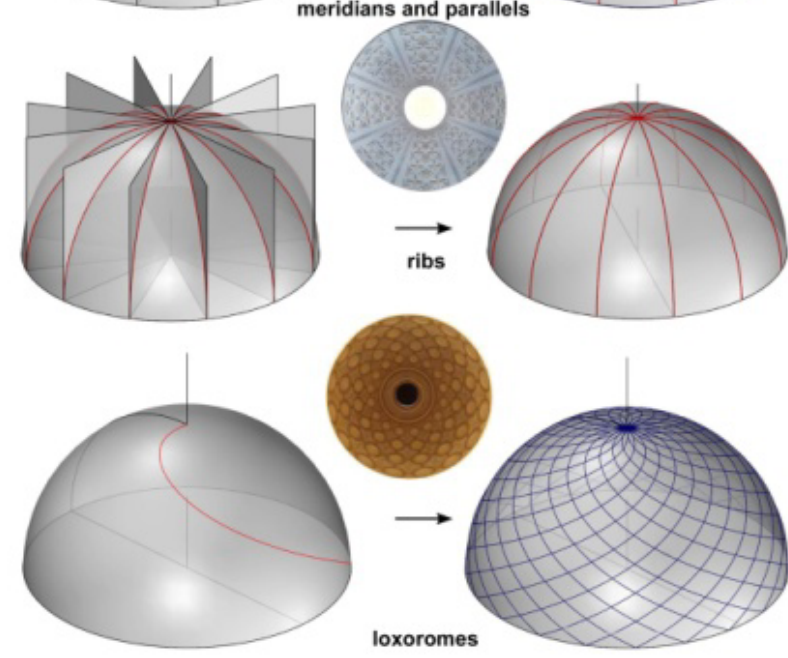

Figure 1. Hemispherical domes: grid geometry for coffers distribution

\footnotetext{
*mara.capone@unina.it
} 

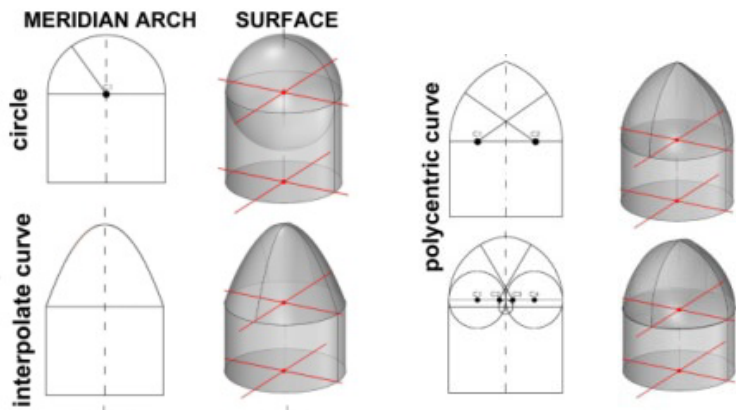

Figure 2. Circular domes: different geometry for domes of revolution

First, we have defined three different way to distribute the coffers in relation to different grid layout (Figure 1):

1. grid composed by 2D lines (meridians and parallels),

2. grid composed by $3 \mathrm{D}$ lines on surface (lattice of rhumb lines, lattice of helices or lattice of Viviani curves)

3. coffers distribution between ribs.

We have analyzed each of them and we have defined different models in relation to coffers shape: quadrilateral, rhomboidal, hexagonal, circular or mixed.

Theoretical models defined according to treaties study have been compared with case studies. This comparison allows us to do a critical analysis based on geometric rules. We are going to study other treatises with the aim of using computational modeling to compare theoretical approach with real architecture.

From a methodological point of view we have built a parametric model able to connect the different processes, using the same parameters. By comparing this model with point clouds, it is possible to evaluate analogies or identify new rules that will be used to develop a more complex parametric model based on surveys.

\subsection{Parametric objects library from historical data}

Parametric tools can reduce human involvement in 3D modeling process, the goal is to develop a pipeline that allows to become this process increasingly automatic. The question is how to check an automatic process without losing the main features of Cultural Heritage. One of the main problems to address in the study and management of the historical heritage is the relationship between the real model, the synthetic geometric model, deriving from the interpretation of big data, and the ideal model.

Generally, the point cloud is semantically decomposed in a $3 \mathrm{D}$ model derived from data simplification: synthetic geometric model. This process is not only automatic, but it is strongly conditioned by the knowledge of the operator or by the analytical tools that the operator has in order to be able to decode historical data. The reverse modeling process, from a point cloud to parametric geometric model, poses a series of issues at the center of cultural debate that currently takes place around HBIM, Historic Building Information Modeling (Paris, 2016). The experimentation underway is part of this research field with the aim of using the parametric approach as a tool able to introduce an additional methodology for big data interpretation.

\subsection{HBIM process}

Currently we can identify two different approaches for HBIM system: building a simplified model by identifying the shape grammar or building the geometric components from survey without using pre-compiled objects libraries, following the scan to BIM logic.

Two problems existed for researchers in generating HBIM, the first is the absence of complex historic architectural elements in BIM libraries and secondly a system for mapping the objects onto remotely sensed survey data (Dore, 2015).

First we have to identify the architectural rules and shape grammars, which we can be applied to develop the library of historical Architectural Elements, this information can be found in various historic architectural texts (Dore, 2015) and then we have to choose the parameters that we can use to modify the object, the procedural rules.

Traditional approach begins with analysis of main data, from the point cloud, which can be used to provide thematic readings. You can operate directly on the cloud, using a color code to associate certain properties, you can highlight parts of the building that have the same characteristics and then add annotations. In this way the points cloud, from an anonymous set of points, acquires a meaning and it could become interface of an information system. Obviously this process is not automatic, just as the transformation of the points cloud into a geometric model divided into elements, each with certain characteristics, is not automatic.

This approach allows to acquire a deep knowledge of the building that goes beyond the visible, with the aim of discovering the founding principles of the construction.

The overlapping of ideal models to $3 \mathrm{D}$ realty based model is a fundamental tool for comparing, analyzing data, processing critical interpretations, identifying rules and understanding exceptions.

\section{ARCHITECTURAL ELEMENTS DEFINITION}

\subsection{Domes shape}

We can define different kind of coffers in relation to coffer shape: square, rectangle, circle, hexagonal, octagon, rhomboidal and mixed configurations. One question that we have to solve is to define the relationships between the coffer shape and the layout used for coffers distribution on the surface and the relationships with the different kind of domes.

Domes are double curvature surfaces, the most common domes are circular base and for that we call them "circular domes", even if their cross-section is not circular (Figure 2). Circular domes are revolution surfaces generated by rotating a meridian curve around a vertical axis. We have identified three kinds of circular domes: domes with meridian curve composed by a quarter of circle, in this case they are "hemispherical domes", domes with meridian curve composed by a pointed arc or polycentric arcs, "pointed domes" and domes generated by parabolic, elliptic or catenary curves (Capone, 2019).

There are domes with elliptical or oval plan, we can group them into ellipsoidal, if they are generated by revolution around an horizontal axis, or ovoidal dome, if they are interpolated surfaces (Figure 4).

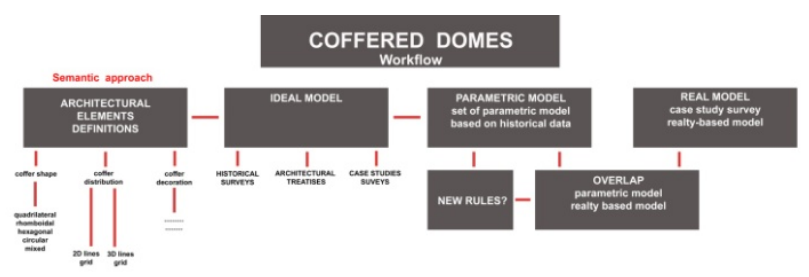

Figure 3. Coffered domes: process for parametric objects library definition 


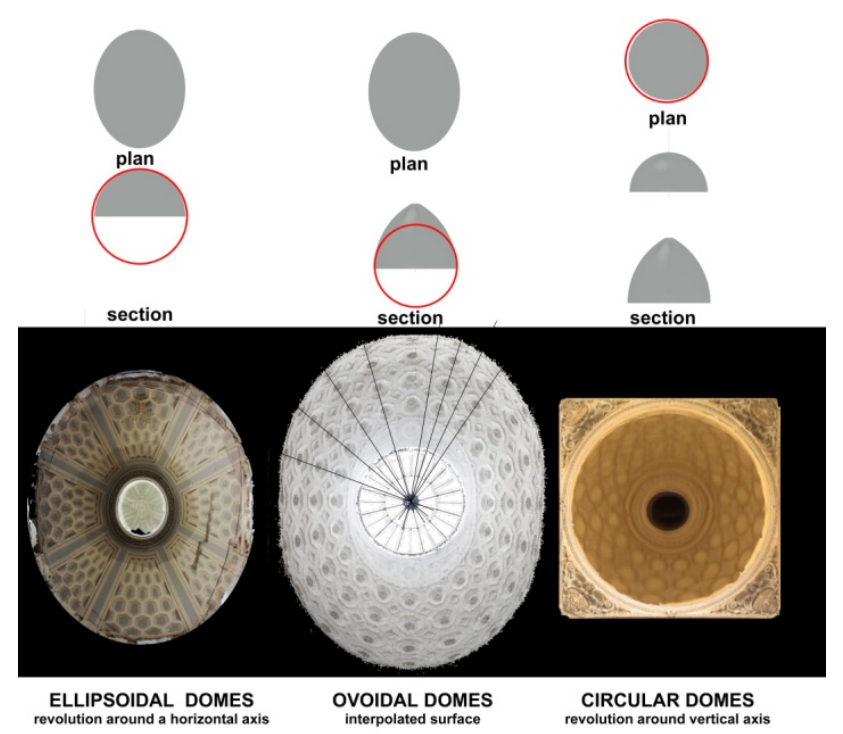

Figure 4. Domes geometry: classification

\subsection{Coffer distribution rules}

We have studied coffer distribution rules starting from one of the most famous coffered dome: Pantheon's dome. It is a circular dome, in particular it is an hemispherical domes and it is characterized by square coffers. There are a lot of hypothesis about the layout for coffers distribution on the dome surface and more of them are related to sphere proprieties and in particular to stereographic projection. The relationships with the dome surface is very important to define the coffers distribution rules. The main parameter that can change in relation to surface is the coffers convergence: they can converge all in the center or not (Figures 14 and 15).

We have defined all these questions related to layout for spherical domes. The main goal of our research is to study what's different in not spherical domes, such as polycentric domes (generated using a polycentric curve that revolves around a vertical axis) or ellipsoidal domes (generated using a polycentric curve or ellipse that revolves around an horizontal axis or ovoidal domes, generated using curves network (Figure 4).

\section{IDEAL MODEL DEFINITION}

There are many differences between the dome real surface and the ideal model. These differences can be caused by structural and constructive movements that occurred over time. Our aims are:

- to determine the original layout hypothesis that could have been used at the moment of construction;

- to identify possible irregularities through a comparative analysis between the real model (from point cloud) and the ideal model (from treatises geometric rules);

- to identify the laws that govern the design of the layout of the dome and coffers in special cases.

From a methodological point of view we have defined the rules for hemispherical domes analyzing historical sources to understand what geometric methods were used in the layout and we have compared these rules with some other kind of surfaces.

\subsection{Geometric rules from treatises}

There are two different approaches to design the web geometry, the layout, on dome surface. The first is a geometric method based only on geometric rules, the second is related to visual perception and the corrections carried out in the layout to improve or direct human perception of architecture (FernándezCabo, 2013). Our aims are to define the geometric rules and the method of perspective or projective geometry used for layout to generate an algorithmic model based on these rules.

The coffered domes can be classified according to the shape of the coffer, that can be quadrilateral, polygonal or circular (Figure 5), or according to grid geometry, that can be composed by meridians and parallels or by no planar lines, such as loxodromes. Furthermore we have identified a further typology of coffered domes in which the grid that defines the large drawers overlaps a partition of the ribbed dome (Figure 1). In the case of the quadrilateral coffers on hemispherical dome the sides of the spherical quadrilateral, which defines the design of the coffer, are circumference arches, if the grid is composed by meridians and parallels, while they are generally loxodromes in the case in which the grid is composed of curves humps. The grid definition by Milizia and Vannini shows that procedures for defining lacunars degradation were linked to projective methods used by the builders of the time rather than by a geometric awareness (Pintore, 2007). Both Vannini and Milizia show procedures that allow us to dimension coffers and ribs using projective methods as a function of the number of "ribs" and without dealing with geometrical configurative genesis of no-planar lines that regulate the distribution, whether they are sides or diagonals of sherical quadrilaterals, centers of circular coffer or sides of spherical quadrilaterals.

However, Vannini manifests need to define a valid geometric method for coffers of any shape and for domes of any curvature, not only hemispherical, and defines a "Rule for coffers rhomboid orthographic draw ... " (Tab. XLIII) (Vannini, 1818) with the aim of avoiding, especially for the inexperienced, strenuous computations aimed only at producing drawings.

Therefore, the objective of our research is to translate the procedures illustrated in treaties, through the comparative study of several sources, to define general geometric rules applies to different cases. The main difficulty lies precisely in identifying the reference parameters in order to obtain a parametric model as flexible as possible.
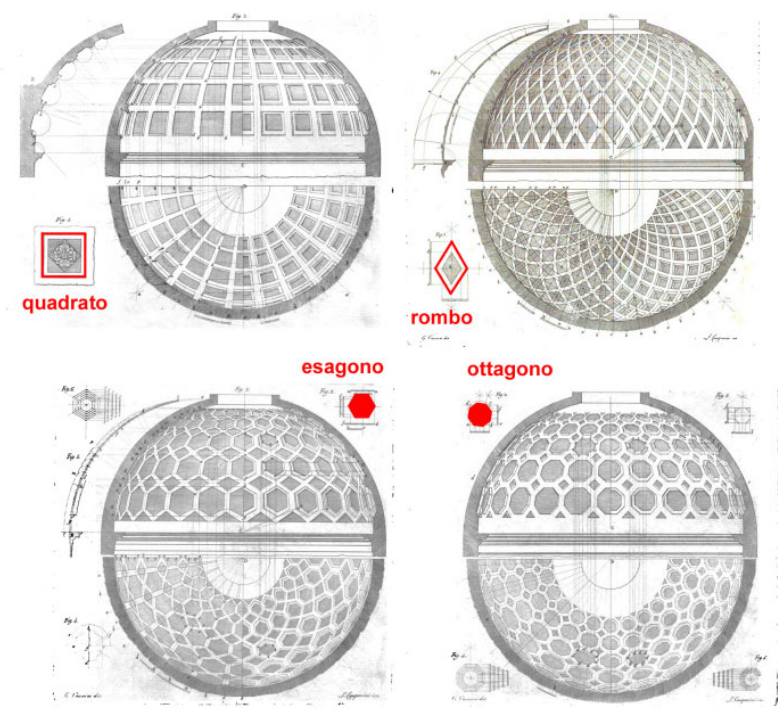

Figure 5. Coffers distribution rules on hemispherical domes by Giuseppe Vannini in "Elementi di Architettura Civile" 


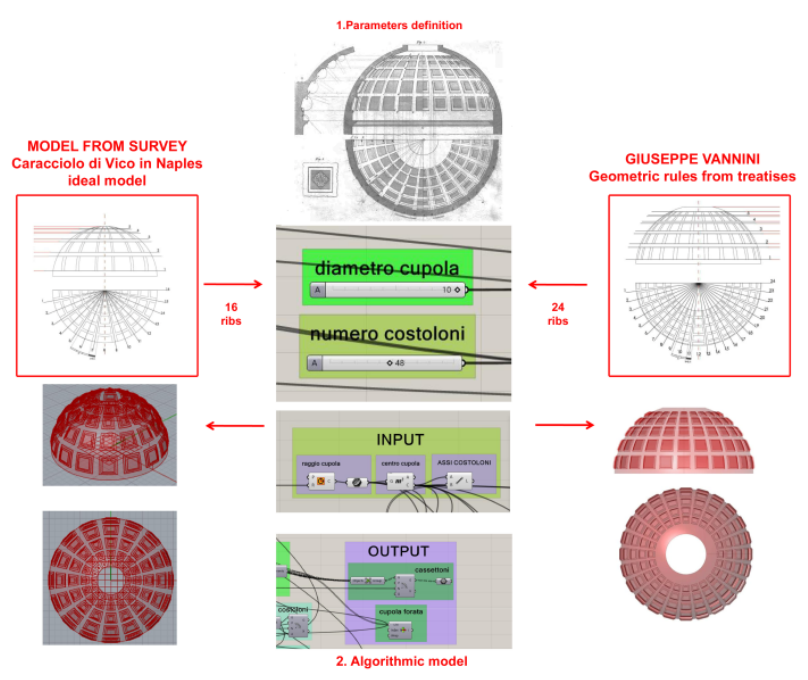

Figure 6. Coffers distribution rules on hemispherical domes by Giuseppe Vannini. Generative model.

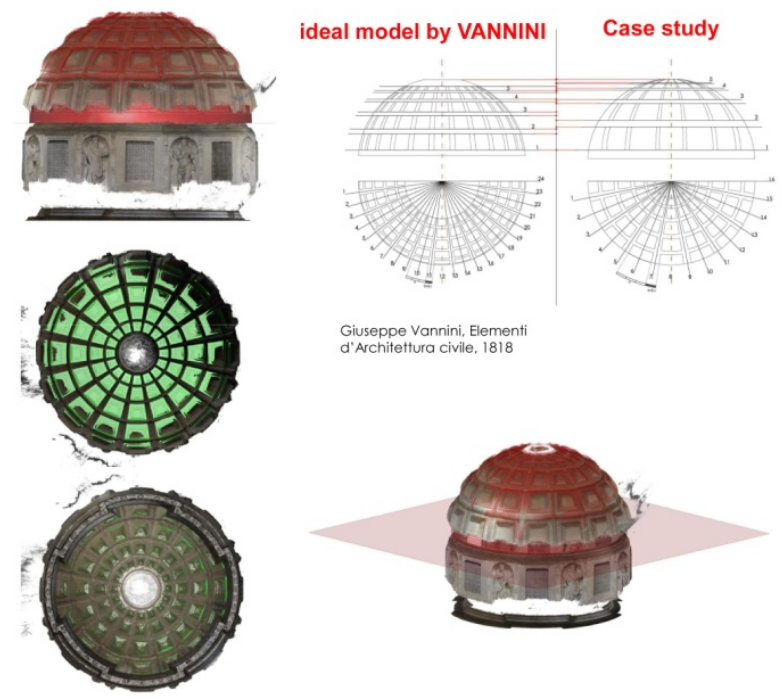

Figure 7. Overlap: parametric model on point cloud of Cappella Caracciolo di Vico in Naples.

The main objective of the research was to experiment with the use of parametric modeling as a tool for critical analysis aimed at the interpretation of big data, point clouds from laser scanner or photogrammetry (Figures 6 and 7). We are going to use procedural modeling techniques to generate HBIM coffered domes library.

Based on the current research problems and the potential of a procedural modeling approach (Dore, 2017) we are working on:

- designing a grammar of parametric shapes and objects that can be used to reconstruct domes geometry from point clouds;

- designing new procedural rules and algorithms for reconstructing domes geometry from point clouds;

- designing efficient methods of interactive editing to alter procedurally generated geometry

- implementing the grammar of shapes, procedural rules and methods for interactive geometry editing using Dynamo or Grashopper;

- using case studies to define new procedural modeling tools.

In order to attempt a 3D model of theoretical project of the circular domes, we need the meridian curve of the revolution surface and to proceed with the layout of the coffered dome, we need:

- the latitude of coffers startups;

- parallel ribs;

- meridian rib;

The grid on dome surface is composed dividing into radial sectors and cutting it using horizontal planes. In this way we obtain spherical trapezoids instead of squares. We can establish that the trapezium is quite close to a square, which leads to the condition that a circle can be inscribed inside, tangent to all sides (Fernández-Cabo, 2013).

To solve this problem we can use the Archimedes theorem on tangent circles and the Apollonio theorem on oblique cone sections (Pintore, 2007).

We have done a parametric model, based on the Archimedes theorem, able to draw tangent circles between two straight lines (radii used to divide plan circle). The input is the number of part in which we divide plan circle and the outputs are the tangent circles (Figure 8). According to Apollonio's theorem we know that the stereographic projection of a given circle on sphere surface is the curve in which the plane of equator intersects the cone with south pole as vertex and the circle as its base. Knowledge of this theorem allows to solve many design problems. Apollonio proved that in an oblique circular cone every subcontrary sections is a circle. To find $\beta$ plane (subcontrary section of circle $\omega$ ) we consider the triangle BAV on $\alpha$ plane, in a point $\mathrm{M}$ we can draw $\mathrm{MN}$ in such a way that the angles $\mathrm{BAV}=\mathrm{NMV}$. $\beta$ is the plane for $\mathrm{MN}$ orthogonal to $\alpha$ (Figure 9), all sections of the cone with planes parallel to $\beta$ are circles (Capone, 2012).

We can prove that the diagonals of spherical quadrilateral, tangent to circles on the sphere surface, are loxodromes because they are the stereographic projection of logarithmic spirals on the equatorial plan (Pintore, 2007).

We have done a parametric model using these geometric rules, the parameters are the dome plan and the number of division. The main steps of the process are (Figures 11, 12 and 13) :

- definition of circles in equatorial plane using Archimede rule;

- stereographic projection of the circles on sphere surface;

- of logarithmic spirals on the equatorial plan;

- stereographic projection of logarithmic spirals

- web geometry definition for coffered distribution.

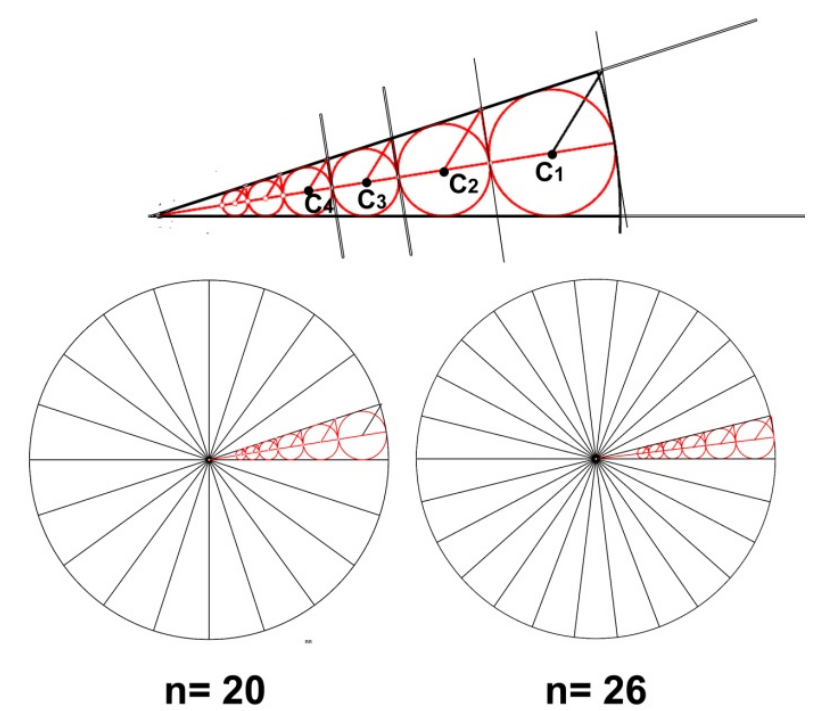

Figure 8. Archimedes theorem on tangent circles: 2D drawing of tangency circles between two (algorithmic model) converging lines. 


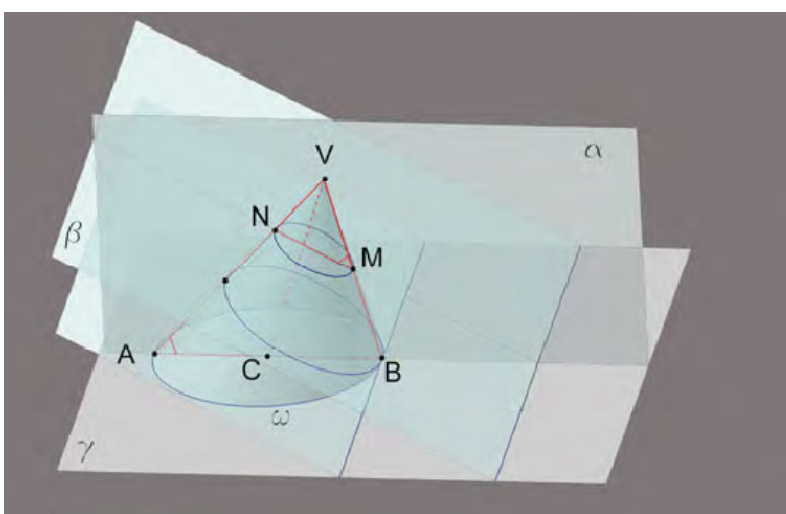

Figure 9. Theorem of Apollonio: sections of an oblique cone

\subsection{Geometric rule from case studies}

One of the main goal of our research is to identify the rules that govern the design of layout coffers in special cases.

We have defined the rules to design the layout for coffered distribution on a spherical domes, the questions are: how do the rules change if the surface is not a spherical surface? What happens if the dome is a surface generated by the rotation of a curve (meridian curve not circle) around a vertical axis? What happens if the dome is a surface generated by the rotation of a curve around a horizontal axis? What happens if the dome is a surface generated using one or more curves that make a closed connection, Patch Surface?

From a methodological point of view, to solve these questions we have selected some case studies and we have carried out a survey.

We have tried to adapt the rules previously defined to the case studies. From the comparison between the point cloud and the theoretical model we are trying to define the general rules adaptable to different cases.

These rules are the basis for generating the parametric model that can be used in an HBIM process.
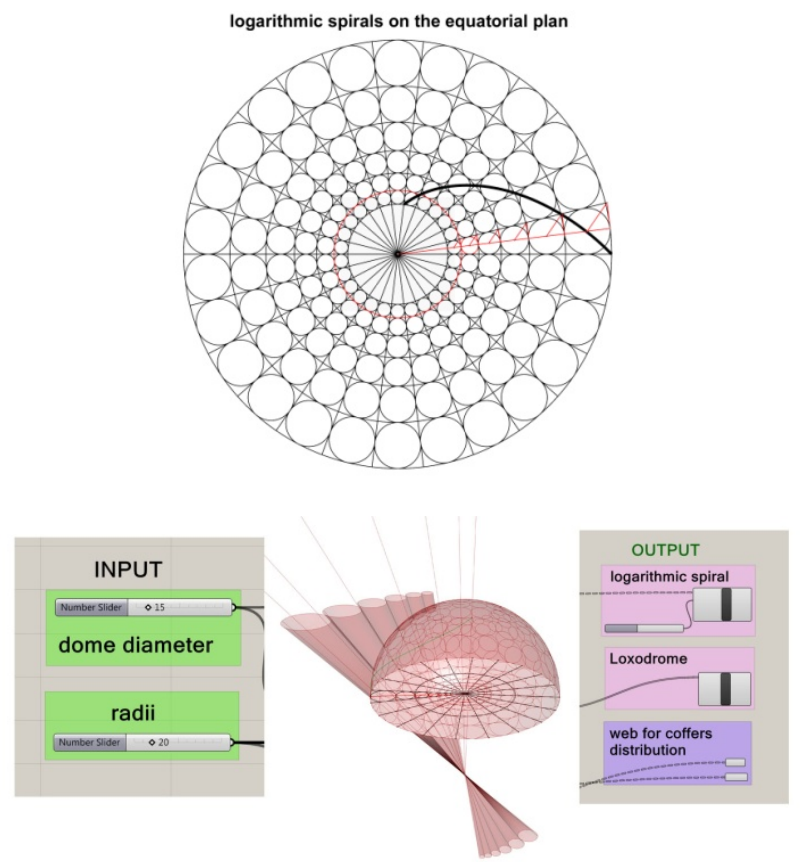

Figure 10. Generative modeling for coffers distribution on hemispherical dome starting to rules by Archimede

\section{PARAMETRIC OBJECT LIBRARY}

Starting from these different hypothesis we have done the following parametric objects based on historical data:

1) based on perspective projection of tangent circles, drawn equator plane, on the surface of the dome;

2) based on central projection of a regular division of the vertical tangent;

3) based on use of $45^{\circ}$ loxodrome.

Our aim is to expand this list using some other rules from historical sources or realty based.

We are going to test a process that can allow us to improve the parametric object library using data from study cases surveys.

We are testing a process based on comparison between ideal model and realty based model to define new rules respect to we can generate new algorithmic models that we can use in the process.

\section{CASE STUDIES}

We have chosen a particularly complex case studies to verify the possibility of using the defined rules, to distribute the coffers on hemispherical domes, even for different surfaces.

In this part of our research we have studied two domes in Naples: the dome of San Giovanni Maggiore, that is an ovoidal dome, and the dome of Padri della Missione, that is an ellipsoidal dome.

First we have verified what kind of surface they are. We hypothesized that the surface was a surface obtained by rotation of the cross section, or of the plan, around the horizontal axis.

We know that the surfaces of rotation enjoy certain properties: the sections orthogonal to the rotation axis are all circumferences (parallel) and the sections obtained with planes passing through the axis are equal to the generating curve (meridian curve). To verify this hypothesis we have generated sections from the cloud of points orthogonal to the horizontal axis (Figures 13 and 14) .

In the dome of $\mathrm{S}$. Giovanni these sections are not circumferences, therefore we have concluded that it is not a surface of revolution.

For this reason we have generated the surface using a network of curves extracted from the point cloud, optimized and adjusted with respect to the principles of symmetry and with the aim of generating an ideal realty based model that does not therefore take into account deformations and any construction defects.

On the contrary, the dome of Padri della Missione is an ellipsoid because the transversal sections are circles.

Once the surface was obtained, we reasoned about the network of curves used for coffers distribution, with the same principles used for hemispherical domes.

The main difference is that in the dome of San Giovanni all coffers converge in the same point, the center, while in the dome of Padri della Missione they converge on a curve.

For this reason we have ruled out the possibility to use a projective method. We are searching the rules for this kind of coffered domes very similar to S. Andrea al Quirinale the most famous dome by Borromini in Rome.

\subsection{Survey}

The reconstruction of precise surfaces from point clouds derived from laser scanner data or photogrammetric image measurements is a very old problem. The generation of polygonal models that can satisfy high modeling and visualization demands is required in different applications. (Remondino, 2013). 


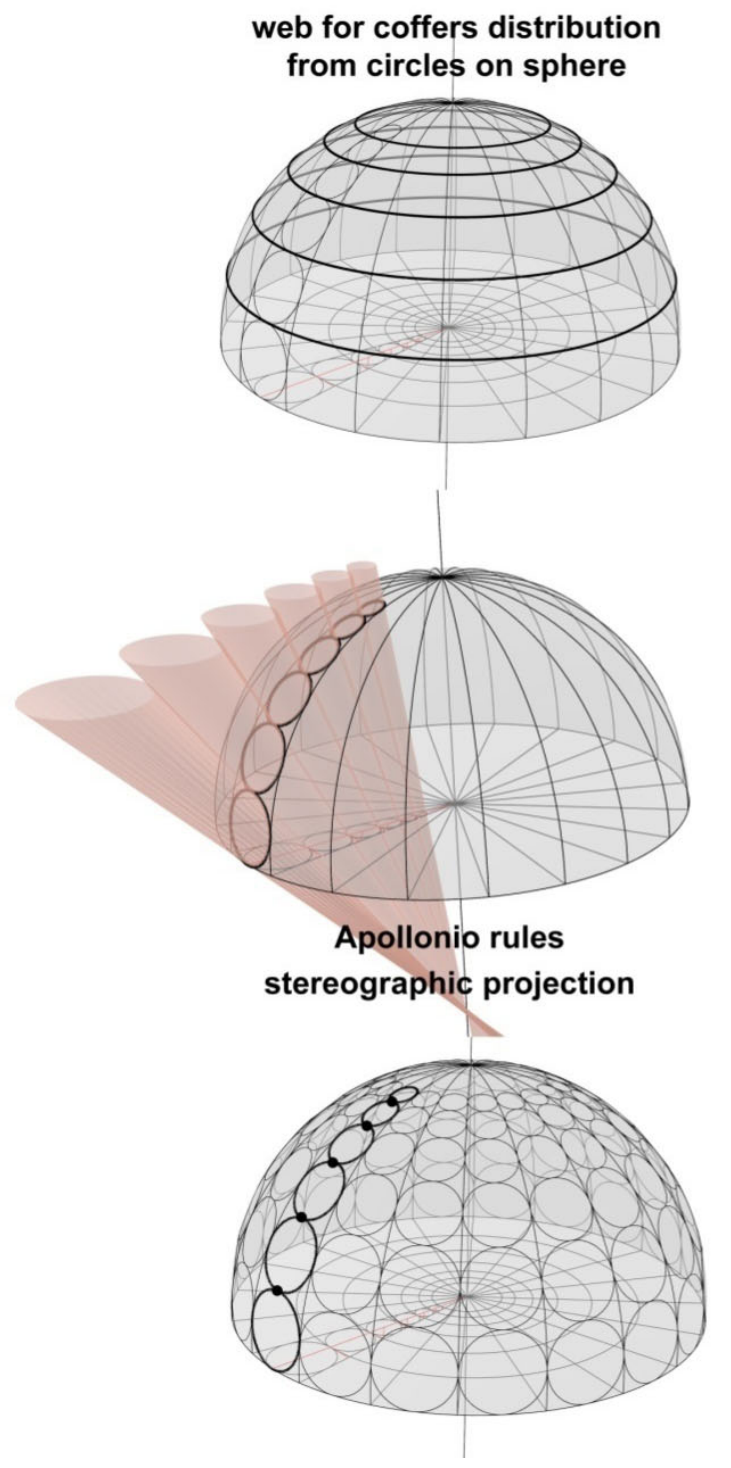

Figure 11. Web for coffers distribution on hemispherical dome staring to Archimede rules and the theorem of Apollonio

There are a lot of papers that face the problem of modeling from point cloud. The reverse modeling is an important issue investigated in many research activities. In Scan to BIM process the main problem that we have to solve is the big data interpretation. There are many studies that propose oriented framework for scan-to-BIM, researchers have developed semiautomated or automated scan-to-BIM techniques to replace the manual approach (Qian, 2019). These developed techniques are focused on the automation of object recognition and geometric modelling of building components from laser scan data.

These developed techniques are focused on the automation of object recognition and geometric modelling of building components from laser scan data (Qian, 2019).

Sometime there are many differences between the real surface and the ideal model, it can depend on constructive reasons or movements that occurred over time. We are interested in understanding the design rules from point cloud rather than to obtain a very accurate 3D model from point cloud.

\section{web for coffers distribution from loxodrome}

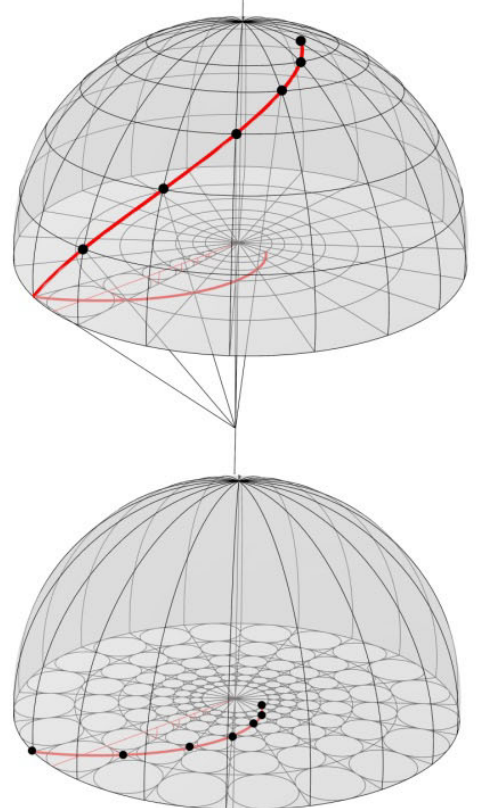

logarithmic spiral

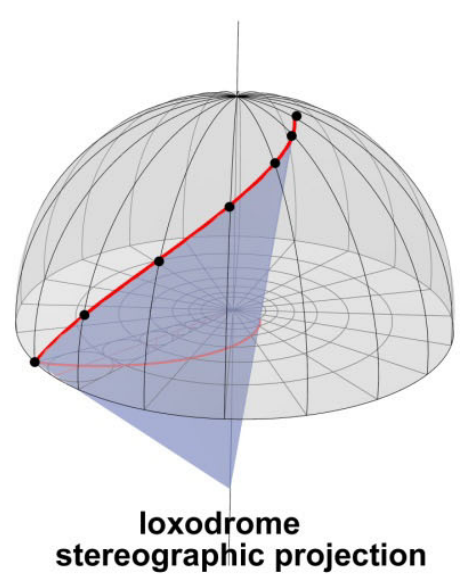

Figure 12. Generative modeling for coffers distribution on hemispherical dome using loxodrome

Our aim is to define a 3D real model (from point cloud) that could reflected the geometric methods used during its conception and methods that were used during its construction. Comparing 3D ideal model and 3D realty based model we can evaluate the differences. The automatic tools to control the level of accuracy.

\subsection{Point cloud interpretation using computational approach}

Our framework for domes geometry definition consists of the following three key steps:

1. generating the $3 \mathrm{D}$ ideal model using the meridian curve from point cloud;

2. generating the $3 \mathrm{D}$ model using curve network from point cloud;

3. comparison between $3 \mathrm{D}$ ideal model and $3 \mathrm{D}$ realty based model. 

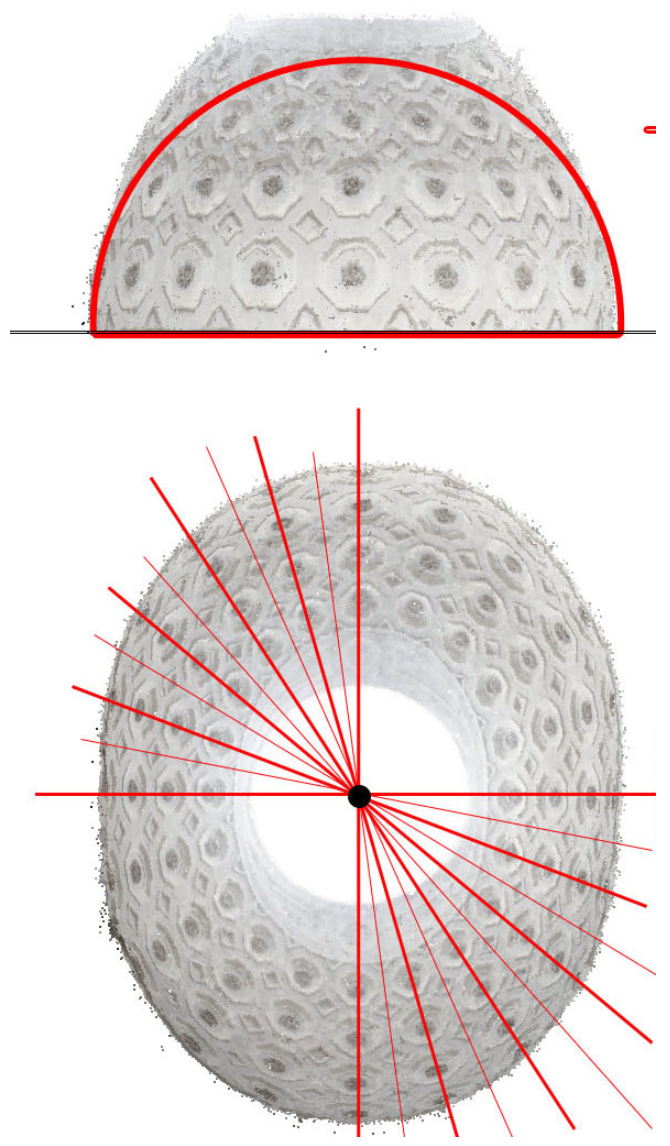

Figure 13. Dome of S.Giovanni Maggiore in Naples. Coffers layout study.

In the case of circular domes, we have done a tool that allows to extract sections from point cloud using computational approach. The process to point cloud interpretation starts from the meridian curves extraction from point cloud. We have defined a meridian curve obtained by comparing all meridian curves extracted.

The 2D polylines extracted from the point cloud are imported in Grasshopper (Rhino, Mc Neel) and they are optimized in continuous curves using a generative algorithmic definition scripting. They are compared each other to define the best curve able to approximate the original design. We can analyse this curve to evaluate what kind of curve it is.

We use this meridian curve to generate the dome surface of revolution: this is the ideal model.

In the case of S. Giovanni Maggiore we have to solve the problem of an interpolated surface because it isn't a surface of revolution. For this reason we have generated 3D realty based model using a curve network extracted from point cloud. To do this we have faced problems about curve network generation from point cloud section, curves optimization, surface generation from curve network. We have generated a Patch Surface using these curve network composed by meridian and parallel lines from point cloud: this is the $3 \mathrm{D}$ realty based model (Figure 15 and 16).

\section{CONCLUSION}

We are working to define a process that can allow us to automatically evaluate the differences between the real model and ideal model.

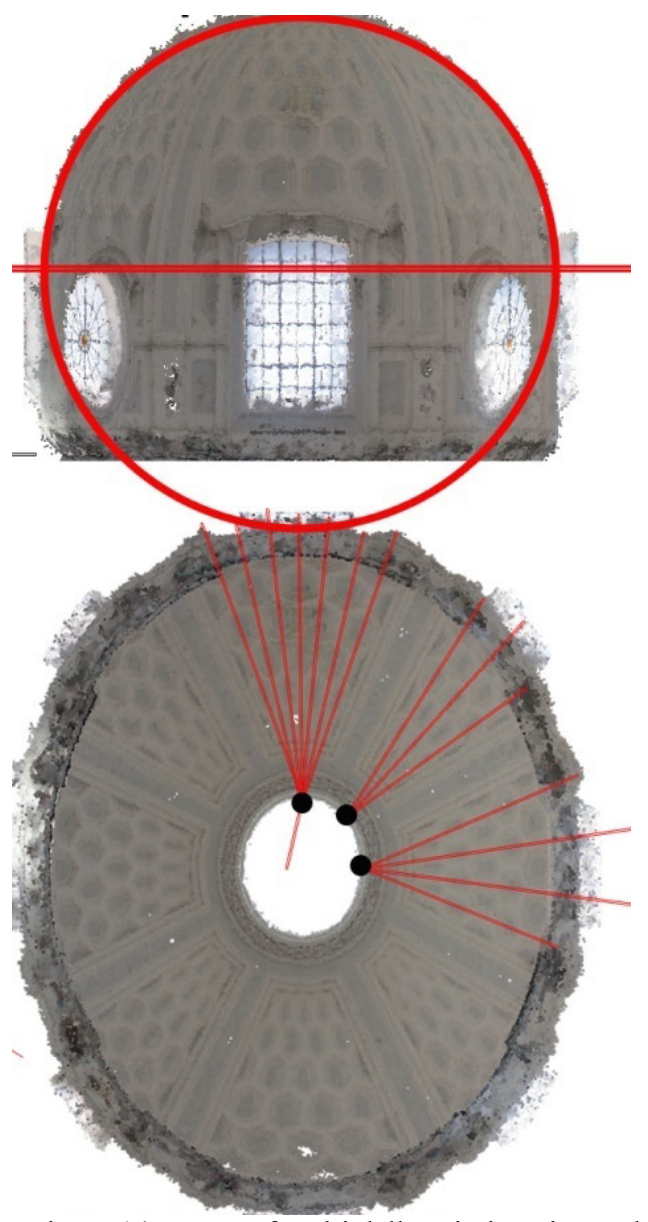

Figure 14. Dome of Padri della Missione in Naples. Coffers layout study.

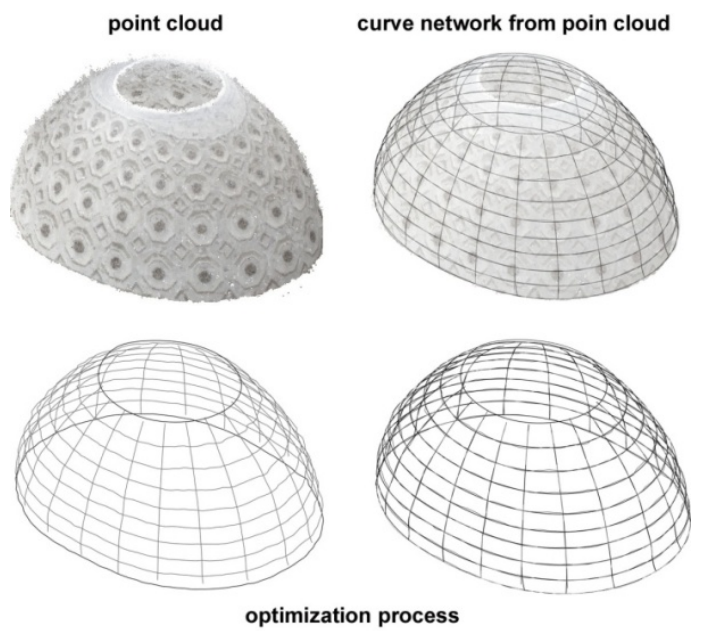

Figure 15. Dome of S. Giovanni Maggiore: ideal model from point cloud

In this paper, we presented a framework for 3D data interpretation using geometric rules from treatises.

The main goal is to generate $3 \mathrm{D}$ ideal model more than a very accurate 3D model from point cloud, our idea is to use this model to note structural problems or some other local anomalies. In the future, we plan to generate a library of algorithmic models for all kind of coffered domes using rules from treatises and rules from survey. 


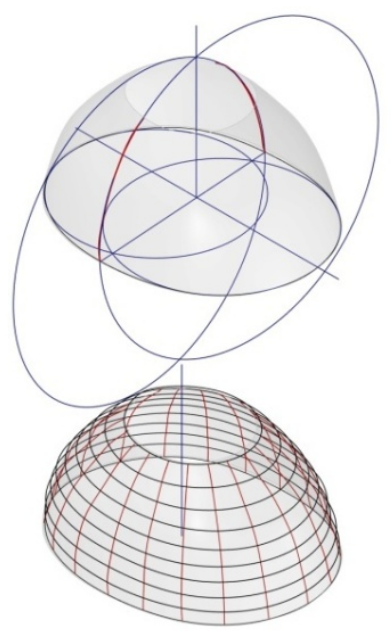

Figure 16. Dome of S. Giovanni Maggiore: ideal model optimization process

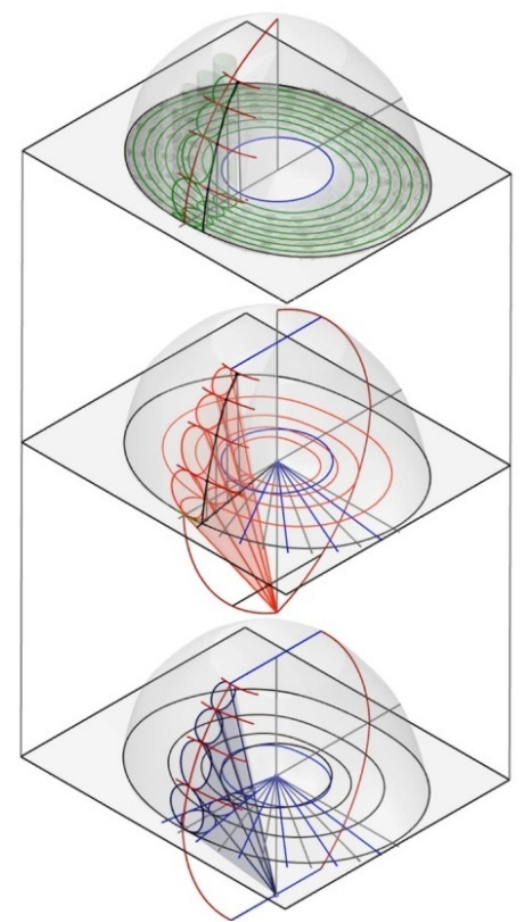

Figure 17. Research in progress: projection hypothesis for ovoidal coffered domes

\section{REFERENCES}

Aliberti, L., Canciani, M., Alonso Rodriguéz, M.A., 2015. New contributions on the dome of the Pantheon in Rome: comparison between the ideal model and the survey model. In: The International Archives of the Photogrammetry, Remote Sensing and Spatial Information Sciences, Volume XL-5/W4, pp.291-297.

Angjeliu, G., Cardani, G., D. Coronelli, 2019. Digital Modelling and analysis of masonry vaults. In: The International Archives of the Photogrammetry, Remote Sensing and Spatial Information Sciences, Volume XLII-2/W11, pp. 83-89.

Attico, D., Turrina, A., Banfi, F., Grimoldi, A., Landi, A., Condoleo, P. and Brumana, R., 2019. The HBIM analysis of the geometry to understand the constructive technique: the use of the trompe volume in a brick vault. In: The International Archives of the Photogrammetry, Remote Sensing and Spatial Information Sciences, Volume XLII-2/W11, pp. 107-114.

Baglioni, L., 2007. Il contributo del modellatore informatico nello studio di lossodromie, eliche e spirali. In: De Carlo, L., 2007. Informatica e fondamenti scientifici della rappresentazione. Gangemi, Roma, pp. 93-102.

Bartoli, M. T., 1994. Scaenographia vitruviana: il disegno delle volte a lacunari tra rappresentazione e costruzione. In: Disegnare. Idee. Immagini, 9/10, pp. 51-62.

Capone, M. 2012. Geometria per l'architettura, Giannini Editore, Napoli, pp.103-104.

Capone, M., Lanzara, E., 2019. Scan to BIM vs 3D ideal model. HBIM: Parametric tools to study domes geometry. In: The International Archives of Photogrammetry, Remote Sensing and Spatial Information Sciences, Vol. XLII-2/W9, pp. 219-226.

Dore, C., 2017. Procedural Historic Building Information Modelling (HBIM) For Recording and Documenting European Classical Architecture. Doctoral Thesis in Built Environment, School of Surveying and Construction Management, Dublin Institute of Technology. doi.org/10.21427/D7045G

Dore, C., Murphy, M., McCarthy, S., Brechin, F., Casidy, C., and Dirix, E., 2015. Structural Simulations and Conservation Analysis -Historic Building Information Model (HBIM). In: The International Archives of the Photogrammetry, Remote Sensing and Spatial Information Sciences, Vol. XL-5/W4, pp. 351-357.

Fernández-Cabo, M. C., 2013. Analysis of Different Hypotheses about the Geometric Pattern of the Pantheon's Coffered Dome. In: Nexus Network Journal, n.15, pp. 527-547. doi.org/10.1007/s00004-013-0165-5.

Migliari, R., 2009. Geometria Descrittiva. Vol. 2. Città Studi, Torino, pp.129-143.

Milizia, F., 1785. Principi di Architettura Civile. Bassano: a spese Remondini di Venezia, pp. 408-410. https://archive.org/details/civilearchitettu01 mili/page/n4 (30 June 2018)

Pintore, A., Salvatore, M., 2007. Shape from points. Morfogenesi e modellazione matematica. In: De Carlo, L., Informatica e fondamenti scientifici della rappresentazione. Gangemi, Roma, pp. 161-174.

Qian, W., Guo, J., Min-Koo, K., 2019. In: Remote Sensing. Vol. 11 issue 3, pp. 1-27. doi.org/10.3390/rs 11030365

Remondino, F., 2013. Fom point cloud to surface: the modeling and visualization problem. In: The International Archives of the Photogrammetry, Remote Sensing and Spatial Information Sciences, Vol. XXXIV-5/W10, pp. 1-11.

Vannini, G., 1818. Elementi di architettura civile per uso degli alunni dell'imperiale e reale accademia delle Belle Arti di Firenze composti e disegnati da Giuseppe Vannini, a spese di Giovacchino Pagani, Firenze,pp 123-126. https://archive.org/details/elementidarchite00vann/page/n4 (30 June 2018) 